\title{
Effects of LPLI on microglial phagocytosis in LPS-induced neuroinflammation model
}

\author{
Sheng Song*, Wei Chen*, ${ }^{* \dagger}$ and Feifan Zhou*** \\ *MOE Key Laboratory of Laser Life Science $\mathcal{E}$ \\ Institute of Laser Life Science \\ College of Biophotonics, South China Normal University \\ Guangzhou 510631, P. R. China \\ ${ }^{\dagger}$ Department of Engineering and Physics \\ University of Central Oklahoma \\ Oklahoma 73034, USA \\ tzhouff@scnu.edu.cn
}

Received 31 May 2013

Accepted 11 September 2013

Published 18 October 2013

\begin{abstract}
Microglial activation plays an important role in neurodegenerative diseases. Once activated, they have macrophage-like capabilities, which can be beneficial by phagocytosis and harmful by secretion of neurotoxins. However, the resident microglia always fail to trigger an effective phagocytic response to clear dead cells or $\mathrm{A} \beta$ deposits during the progression of neurodegeneration. Therefore, the regulation of microglial phagocytosis is considered a useful strategy in searching for neuroprotective treatments. In this study, our results showed that low-power laser irradiation (LPLI) $\left(20 \mathrm{~J} / \mathrm{cm}^{2}\right)$ could enhance microglial phagocytic function in LPS-activated microglia. We found that LPLI-mediated microglial phagocytosis is a Rac-1-dependent actin-based process, that a constitutively activated form of Rac1 (Rac1Q61L) induced a higher level of actin polymerization than cells transfected with wild-type Rac1, whereas a dominant negative form of Rac1 (Rac1T17N) markedly suppressed actin polymerization. In addition, the involvement of Rac1 activation after LPLI treatment was also observed by using a Raichu fluorescence resonance energy transfer (FRET)-based biosensor. We also found that PI3K/Akt pathway was required in the LPLI-induced Rac1 activation. Our research may provide a feasible therapeutic approach to control the progression of neurodegenerative diseases.
\end{abstract}

Keywords: Microglia; phagocytosis; LPLI; Rac1.

This is an Open Access article published by World Scientific Publishing Company. It is distributed under the terms of the Creative Commons Attribution 3.0 (CC-BY) License. Further distribution of this work is permitted, provided the original work is properly cited. 


\section{Introduction}

Microglial cells constitute the resident professional macrophage population of the central nervous system (CNS). They perform homoeostatic activity and mediate the innate defense system in the normal CNS. However, localized activation of microglia has been implicated in the pathogenesis of several neurodegenerative disorders, such as Parkinson's disease (PD), Alzheimer's disease (AD) and multiple sclerosis. ${ }^{1} \mathrm{AD}$ is characterized by the progressive deposition of $\beta$-amyloid (A $\beta$ ) in the extracellular space of the brain and its pathogenesis is associated with alteration in $\mathrm{A} \beta$ homeostasis, resulting in the accumulation of $\mathrm{A} \beta$ peptides within the brain parenchyma. $^{2}$

The role of fibrillar $\mathrm{A} \beta$ in microglial activation in $\mathrm{AD}$ has been well established. Once microglia are activated in neurodegenerating microenvironment, they have macrophage-like capabilities, including phagocytosis and production of inflammatory cytokine. $^{3}$ Activated microglia can phagocytose fA $\beta$ or dead cells from CNS and can secrete different neurotrophic factors for neuronal survival; however, the resident microglia always fail to trigger an effective phagocytic response to clear $\mathrm{A} \beta$ deposits during $\mathrm{AD}$ progression. ${ }^{4}$ Once activated, they eventually become more detrimental by releasing proinflammatory molecules (NO and TNF- $\alpha$ ). ${ }^{5}$ Therefore, activation of microglia has become a hallmark of AD. It has been debated whether neuroinflammation is an underling cause or a resulting condition in $\mathrm{AD}$.

Low-power laser irradiation (LPLI) can modulate a broad spectrum of cellular processes ranging from proliferation to apoptosis. It has been reported that the effects of laser irradiation on cell proliferation or inhibitory are related to light fluence. ${ }^{6,7}$ This phenomenon of photobiomodulation has also been widely applied in the treatment of skeletal muscle regeneration, ${ }^{8}$ wound healing ${ }^{9}$ and skin wound care. ${ }^{10}$ Reports have shown that $\mathrm{A} \beta$-induced cell apoptosis was significantly diminished with light irradiation. ${ }^{11}$ Besides, the preventive effects of LPLI on $\mathrm{A} \beta_{25-35}$-induced cell apoptosis have been demonstrated, by promoting Akt-dependent YAP cytoplasmic translocation. ${ }^{12}$

Rho GTPases regulate actin cytoskeleton organization in various cell types, including macrophages, by cycling between GDP-bound inactive and GTP-bound active conformations. ${ }^{13-15}$ Rac1 is a Rho-family GTP-binding protein that controls lamellipodia formation and membrane ruffling in fibroblasts. Since microglia can be beneficial by phagocytosing $\mathrm{A} \beta$ or harmful by secretion of neurotoxins, we reasoned that Rac1 may be involved in microglial functional regulation under LPLI. Thus, the effect of LPLI on microglia functions needs to be clarified in developing strategies to slow or prevent the progression of $\mathrm{AD}$.

In this study, we investigated the effects of LPLI on LPS-activated microglia-mediated phagocytic function neurotoxicity using microglia-like BV-2 cells. Our results suggested that LPLI could induce Rac1 activation for neuroprotection by enhancing phagocytic activity.

\section{Materials and Methods}

\subsection{Chemicals and plasmids}

The following reagents were used: lipopolysaccharide (LPS) purified from Salmonella typhimurium (Sigma-Aldrich, St. Louis, MO) to stimulate microglia, wortmannin and API-2 (Sigma-Aldrich) to inhibit phosphatidylinositol 3-kinase (PI3K), API-2 (Sigma-Aldrich) to inhibit Akt, SMT $\left(\left(\mathrm{C}_{2} \mathrm{H}_{6} \mathrm{~N}_{2} \mathrm{~S}\right)_{2}\right.$. $\mathrm{H}_{2} \mathrm{SO}_{4}$ ) (Sigma-Aldrich) to inhibit iNOS, FITCphalloidin to stain F-actin, latex bead to detect microglial phagocytosis.

In addition, we used Lipofectamine LTX with PLUS reagent (Invitrogen, Carlsbad, CA, USA) to transfect plasmid DNA into cells and the cells were examined 36-48 $\mathrm{h}$ after transfection. The plasmid of pRaichu-Rac1 was kindly supplied by Dr. Michiyuki Matsuda. Rac1Q61L, Rac1T17N and wt Rac1 were purchased from Upstate Biotechnology (Lake Placid, NY).

Anti-pAkt (ser473), anti-Akt, anti-Rac1 and anti- $\beta$-actin were all purchased from Cell Signaling Technology (Beverly, MS, USA).

\subsection{Cell culture}

Murine microglia-like cell line BV-2 was maintained in Dulbecco's modified Eagle's medium (GIBCO), with $15 \%$ heat-inactivated fetal calf serum (FCS), penicillin $(100$ units $/ \mathrm{ml})$ and $(100 \mu \mathrm{g} / \mathrm{ml})$ in $5 \%$ $\mathrm{CO}_{2}, 95 \%$ air at $37^{\circ} \mathrm{C}$ in a humidified incubator. To generate activated microglia, cells were stimulated with LPS alone $(100 \mathrm{ng} / \mathrm{ml})$ or with different concentrations of inhibitors before LPLI treatment. 


\subsection{LPLI treatment}

The experiment was conducted as described in our previous work. ${ }^{16}$ After $36-48 \mathrm{~h}$ of serum starved with $0.5 \%$ fetal bovine serum (FBS), the BV-2 cells were irradiated with $\mathrm{He}-\mathrm{Ne}$ laser $(632.8 \mathrm{~nm}$, spot diameter $0.635 \mathrm{~cm}$; HN-1000, Guangzhou, China) for $5.32 \mathrm{~min}$ in the dark, with the corresponding fluences of $20 \mathrm{~J} / \mathrm{cm}^{2}$, respectively (light guide tip output $25.2 \mathrm{~mW}$, power density $64.6 \mathrm{~mW} / \mathrm{cm}^{2}$ ).

\subsection{Transient transfection}

Transient transfection of cells was performed using FuGENE HD transfection reagent following the manufacturer's instructions.

\subsubsection{Laser confocal scanning microscopy (LCSM) and florescence resonance energy transfer (FRET) analysis}

Fluorescent emission from fluorescein isothiocyanate (FITC), and latex beads were monitored with LCSM (Zeiss, Jena, Germany), using different excitation wavelengths and detection filters as previously described. ${ }^{17}$

FRET was performed on LCSM to detect the interaction between cyan fluorescent protein (CFP) and yellow fluorescent protein (YFP). For excitation, the 458-nm line of an argon-ion laser was attenuated with an acousto-optical tunable filter, reflected by a dichroic mirror (main beam splitter HFT458), and focused through a Plan-Neofluar $40 \times / 1.3$ NA oil DIC objective (Carl Zeiss) onto the sample. CFP and YFP emissions were collected through 470- to 500- and 535- to 545-nm band-pass filters, respectively. The quantitative analysis of the fluorescence images was performed using Zeiss Rel3.2 image processing software (Carl Zeiss). FRET was corrected by subtracting the bleed-through signals of the donor and the acceptor.

\subsection{Cell viability assay}

Cell viability was determined using a CCK-8 cell viability assay kit according to the manufacturer's instructions. All cells $\left(5 \times 10^{3}\right.$ cells/well $)$ were pretreated with various methods as indicated and then incubated for $24 \mathrm{~h}$ in a 96 -well plate. A $10 \mu \mathrm{l}$ of cell viability assay kit solution was added to each well of the plate. After incubation for $1 \mathrm{~h}$ at $37^{\circ} \mathrm{C}$ in the dark, absorbances were measured at $450 \mathrm{~nm}$ using a multiwell plate reader.

\subsection{Phagocytosis assay}

Microglial BV-2 cells were collected and $1 \times 10^{5}$ cells were cultured in $35-\mathrm{mm}$ glass-bottomed dishes overnight. The cells were incubated in the presence or absence of the inhibitors, then subjected to LPLI treatment. The fluorescent microspheres, as a marker of phagocytosis, were then added to the treated cells for indicated time periods after having been washed in PBS containing 0.1\% BSA. Cells were then fixed with $4 \%$ paraformaldehyde, and three random fields of cells ( $>100$ cells) were counted under confocal microscope.

Phagocytic efficiency was determined by referring to Pan et al. ${ }^{18}$ Briefly, the phagocytic efficiency was based on a weighted average of ingested microspheres per cell. The number of cells containing microspheres, the number of microspheres per cell and the total number of cells were counted, respectively. Phagocytic efficiency $(\%)=(1 \times X 1+$ $2 \times X 2+3 \times X 3 \cdots+n \times X n) /$ the total number of cells $\times 100 \%$. $X n$ represents the number of cells containing $n$ microspheres $(n=1,2,3, \ldots$, up to a maximum of 6 points for more than 5 microspheres ingested per cell).

\subsection{Phalloidin staining}

The treated cells were rinsed with PBS before being fixed in $3.7 \%$ formaldehyde and washed again in PBS. The cells were then incubated at room temperature with $0.1 \%$ Triton X-100 buffer for $5 \mathrm{~min}$ and washed again in PBS. FITC-phalloidin (1:50 diluted in PBS) was added to the coverslips and incubated at room temperature protected from light for $30 \mathrm{~min}$. The coverslips were mounted on glass slides. The association of FITC-labeled phalloidin was viewed under confocal microscope.

\subsection{Western blot analysis}

Expressions of proteins were quantified by western blot analysis. After individual incubations, cell proteins were extracted in lysis buffer $(50 \mathrm{mM}$ Tris$\mathrm{HCl} \mathrm{pH} 8.0,150 \mathrm{mM} \mathrm{NaCl}, 1 \%$ TritonX-100, $100 \mu \mathrm{g} / \mathrm{ml}$ phenylmethanesulfonyl fluoride (PMSF)) supplemented with protease inhibitor coocktail 
set I for $60 \mathrm{~min}$ on ice. After centrifugation $\left(4^{\circ} \mathrm{C}\right.$, $12,000 \mathrm{rpm}, 20 \mathrm{~min})$, the resulting lysates were resolved on SDS-PAGE Bis-Tris gels $(30 \mathrm{mg} /$ lane; Invitrogen, Life Technologies, Grand Island, NY) and transferred to polyvinylidene fluoride (PVDF) membranes (Millipore, Bedford, MA). The membranes were blocked in TBST $(10 \mathrm{mM}$ Tris- $\mathrm{HCl}, \mathrm{pH}$ 7.4, $150 \mathrm{mM} \mathrm{NaCl}, 0.1 \%$ Tween-20) containing $5 \%$ nonfat milk and then incubated with a designated primary antibody and a secondary antibody. The signals were detected with an ODYSSEY Infrared Imaging System (Li-Cor, Lincoln, NE). The intensity of the western blot signals was quantitated using ImageJ software (NIH, Bethesda, MD).

\subsection{Quantitative measurement of F-actin}

To assess the total F-actin content in BV-2 cells, microglia were treated with LPS for indicated time periods. The reaction was stopped by the addition of formaldehyde $(3.7 \%$ final, $\mathrm{V} / \mathrm{V})$ for $15 \mathrm{~min}$ at room temperature. The fixed cells were then permeabilized with $10 \mathrm{mM}$ imidazole, $40 \mathrm{mM} \mathrm{KCl}$, $10 \mathrm{mM}$ ethylene glycol tetraacetic acid (EGTA), $1 \mathrm{mM} \mathrm{MgCl}$, and $1 \%$ Triton $\mathrm{X}-100$ at $4{ }^{\circ} \mathrm{C}$ for 15 min. F-actin was then stained with FITC-phalloidin (Molecular Probes) for $2 \mathrm{~h}$ at room temperature. After the cells were washed with PBS, F-actinbound FITC-phalloidin was extracted with methanol. The extracts were centrifuged to remove any insoluble material, and relative fluorescence was measured using a 96-well plate reader with excitation and emission wavelengths set at 465 and $535 \mathrm{~nm}$, respectively. The F-actin ratio was calculated as (F-actin in treated cells - background)/ (F-actin in indicated cells - background).

\subsection{Rac1 activity assay}

We measured Rac1 activity in LPLI-treated microglial cell lysate using a kit (Rac1 Activation Assay Kit; Upstate Biotechnology) according to the manufacturer's instructions.

\subsection{Statistical analysis}

All data are expressed as mean \pm SEM. Student's $t$-test was used for all statistical analysis. Values of $P<0.05$ were considered to be statistically significant.

\section{Results}

\subsection{Effects of LPLI on LPS-activated microglial phagocytic activity}

Given that phagocytosis is the process which results in the uptake of large particles $(\geq 1 \mu \mathrm{m})$ by an actin-based mechanism, we then ask whether LPLI could enhance microglial phagocytic activity. Microglial phagocytosis was monitored by the internalization of fluorescent microspheres. Such microbeads have been used previously to investigate signaling in phagocytic cells. ${ }^{19}$

For phagocytosis analysis, a control group (LPStreated group) subtraction was performed. Exposure of BV-2 cells to LPLI at a dose of $20 \mathrm{~J} / \mathrm{cm}^{2}$ for $30 \mathrm{~min}$ produced the maximal phagocytic response, in which the relative percentage of phagocytosed cells and that of phagocytic efficiency were $34.03 \pm 5.1 \%$ and $145.48 \pm 17.1 \%$, respectively. Since actin assembly provides the driving force for particle engulfment by allowing the extension of membrane pseudopods that wrap the particle and eventually close to form a phagosome, we also observed F-actin recruitment and cupping around beads in BV-2 cells. LPLI markedly enhanced microglial phagocytosis, as shown in Fig. 1(a).

To determine the effect of LPLI on LPS-activated microglial viability, the cells were quantitated using a CCK-8 cell counting kit. As shown in Fig. 1(b), LPS clearly suppressed the microglial viability, while the relative cell viability was increased after LPLI treatment. These data suggested that LPLIinduced enhanced microglial phagocytosis might be associated with the changes in cells' viability.

\subsection{Actin-based phagocytosis is a Rac1-dependent process}

Rac1 activation, by cycling between GDP-bound inactive and GTP-bound active conformations, has been shown to induce actin polymerization and is essential for cell phagocytosis. ${ }^{20-22}$ We explored the role of Rac1 in LPLI-mediated microglial phagocytosis. It is clearly evident as shown in Fig. 2(a) that a constitutively activated form of Rac1 (Rac1Q61L)induced actin polymerization was much more than cells transfected with wild-type Rac1, whereas a dominant negative form of Rac1 (Rac1T17N) markedly suppressed actin polymerization.

To further study the effects of Rac1 activity on actin polymerization, we quantified $\mathrm{F}$-actin content 


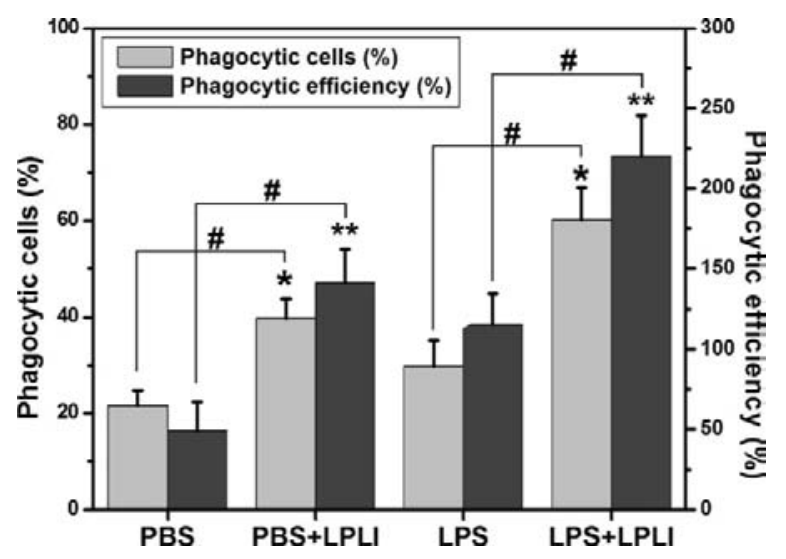

(a)

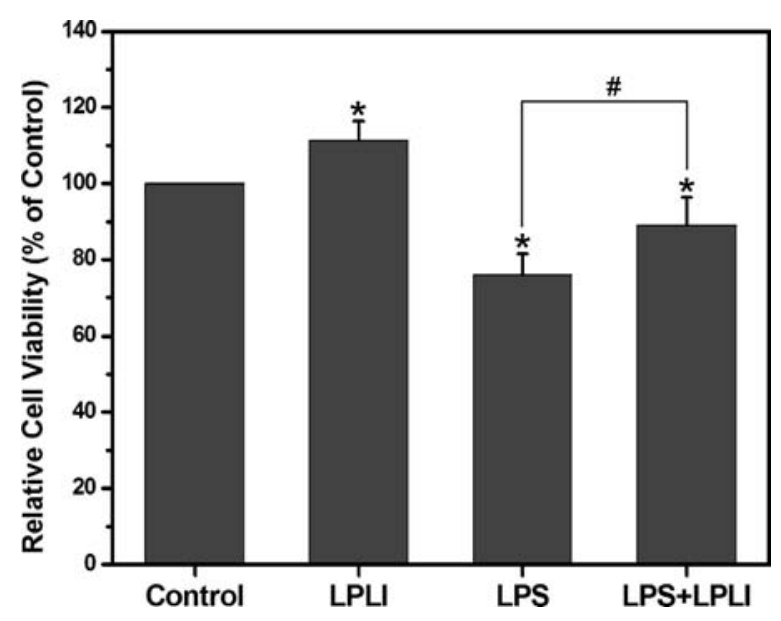

(b)

Fig. 1. Effects of LPLI on phagocytic function of activated microglial cells. BV-2 cells with or without LPLI $\left(20 \mathrm{~J} / \mathrm{cm}^{2}\right)$ treatment were incubated with microspheres for indicated time. (a) Quantification of LPLI-mediated microglial phagocytosis; and the number of microspheres taken up per BV-2 cell at 30 min was measured. ( $n=4 ;{ }^{*} p<0.05$ and ${ }^{* *} p<0.05$ vs corresponding control cells, $\# p<0.05$ vs indicated cells). (b) Quantification of cell viability by using CCK-8 assay after indicated treatments. $\left(n=6\right.$; ${ }^{*} p<0.05$ vs corresponding control cells, $\# p<0.05$ vs indicated cells).

in LPS-activated BV-2 cells that were transfected with different Rac1 constructs under LPLI treatment [see Fig. 2(b)]. The results were consistent with the observation in Fig. 2(a).

To further explore the involvement of Rac1 activation after LPLI treatment, we adopted the FRET technique to investigate Rac1 activation after LPLI treatment. BV-2 cells were transfected with Raichu-Rac1, and then the real-time CFP, FRET and FRET/CFP fluorescence images were collected with LCSM. The results showed that the interaction of CFP and YFP increased under LPLI treatment [see Figs. 2(c)-2(d)], indicating that activation of Rac1 is involved in this process.

\subsection{LPLI-induced Rac1 activation is mediated by PI3K/Akt}

Activation of PI3K/Akt signaling pathway has been correlated with macrophages phagocytosis. ${ }^{23}$ To address whether Rac1 activation is mediated by PI3K/Akt signaling pathway, LPS-activated BV-2 cells were pre-treated with wortmannin, which covalently inactivates the PI3K catalytic site, and API-2, an Akt inhibitor. As shown in Fig. 3(a), the phosphorylation level of Akt was increased after LPLI treatment. Both wortmannin and API-2 markedly reduced Rac1 activity after LPLI treatment [see Fig. 3(a)]. These results suggest that
LPLI induces Rac1 activation in a PI3K/Akt signaling pathway-dependent manner.

To determine whether the inhibition of PI3K/ Akt signal in microglial cells prevents actin polymerization, we measured microglial phagocytosis in BV-2 cells that were treated by LPS with or without wortmannin or API-2 after LPLI treatment. The presence of wortmannin or API-2 inhibited the F-actin accumulation and markedly suppressed BV-2 microglial phagocytosis [see Figs. 3(b) and 3(c)]. Taken together, our results suggest that PI3K/Akt pathway is required in the LPLI-induced Rac1 activation.

\section{Discussion}

Rac1 has two different roles in the engulfment of apoptotic cells. The first is to mobilize plasma membranes and establish lamellipodia, and the second is to regulate the actin dynamics during engulfment. ${ }^{24}$ In the present study, we found a regulatory role of LPLI for microglial functions. The major findings showed that microglial phagocytosis is improved after LPLI treatment, characterized by enhanced Rac1 activity, actin polymerization and the ability of phagocytosis of the microbeads. Moreover, we found that LPLI could induce the enhancement of microglial phagocytic activity by a PI3K/Akt-mediated Rac1-dependent pathway. 


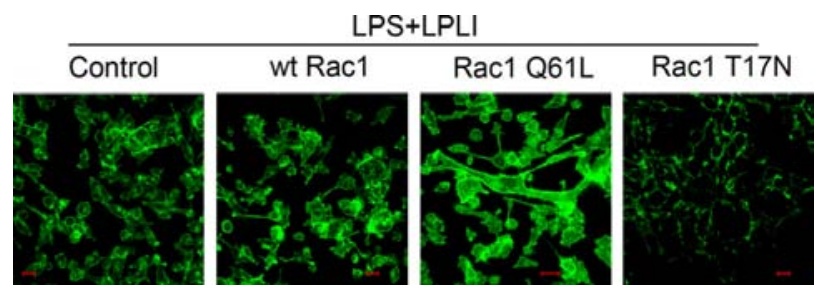

(a)

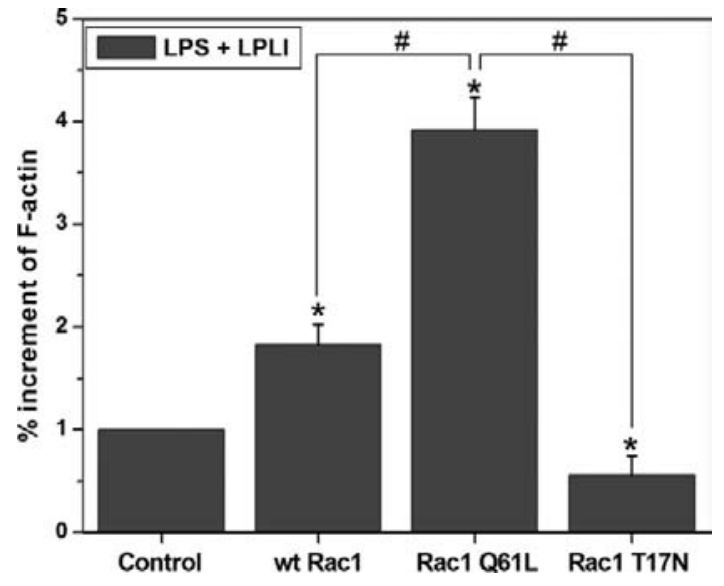

(b)
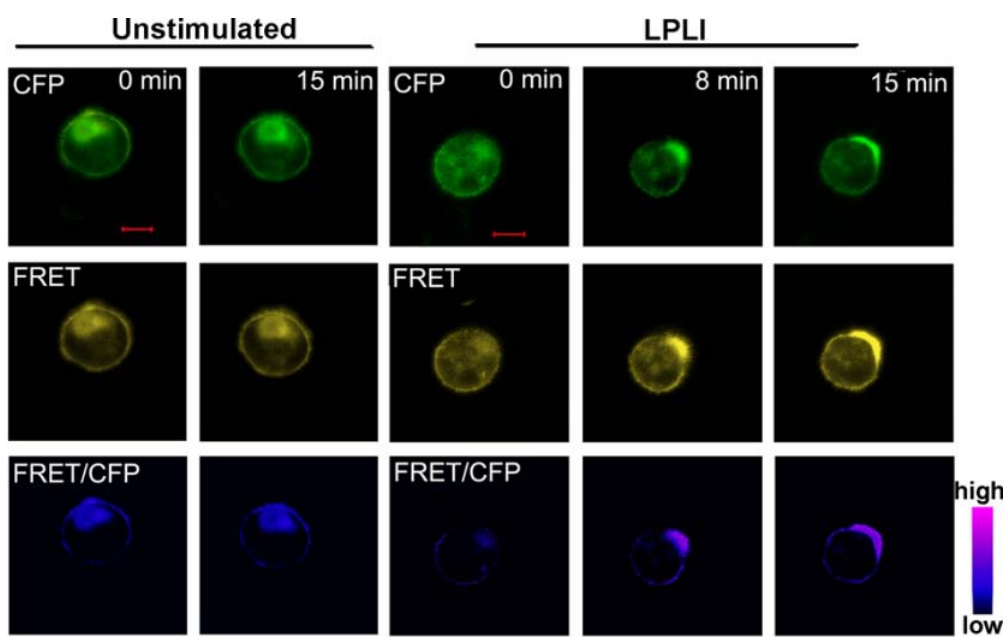

(c)
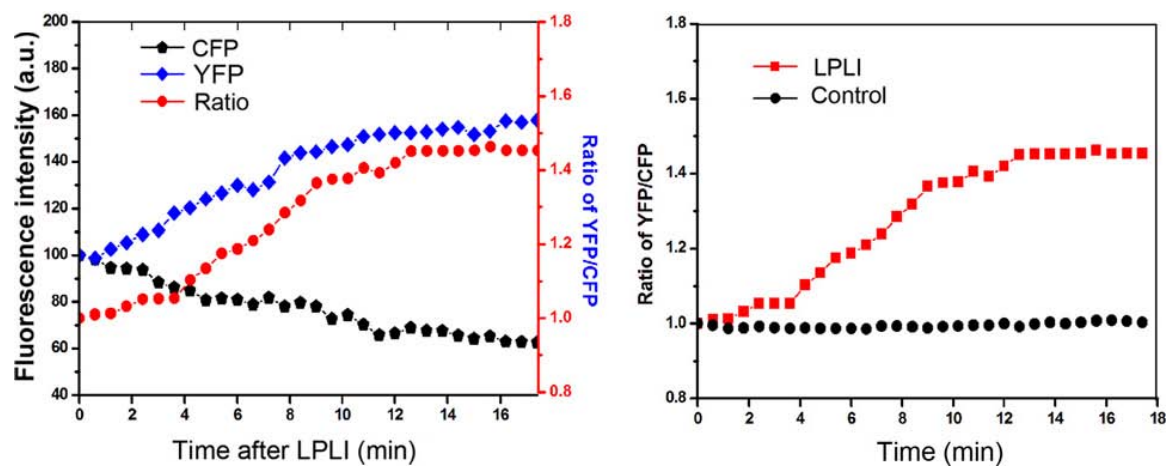

(d)

Fig. 2. LPLI-mediated microglial phagocytosis is a Rac-1-dependent actin-based process. (a) BV-2 cells were transfected with Rac1Q61L, Rac1T17N or wt-Rac1; G418-resistant cells were collected for further LPS stimulation. About 30 min after LPLI (20 J/ $\mathrm{cm}^{2}$ ) treatment, cells were fixed with $4 \%$ paraformaldehyde and stained with FITC-labeled phalloidin to visualize F-actin (green) and confocal Z-stacks were acquired. Images shown are representative of approximately 100 cells. Bar $=50 \mu \mathrm{m}$. (b) Quantitative analysis of the levels of F-actin in (a). Data represent mean $\pm \operatorname{SEM}(n=6)$. (c) Representative fluorescence image series of CFP, FRET and FRET/CFP in control (left panel) and LPLI treated Raichu-Rac1 transfected microglia (right panel). The images of FRET/CFP ratio were processed with pseudocolor technique. Bar $=10 \mu \mathrm{m}$. (d) Quantitative analysis of FRET/CFP ratio corresponding to the images in (c). The FRET/CFP ratio is normalized to 1. Results represent one of five replicates. 


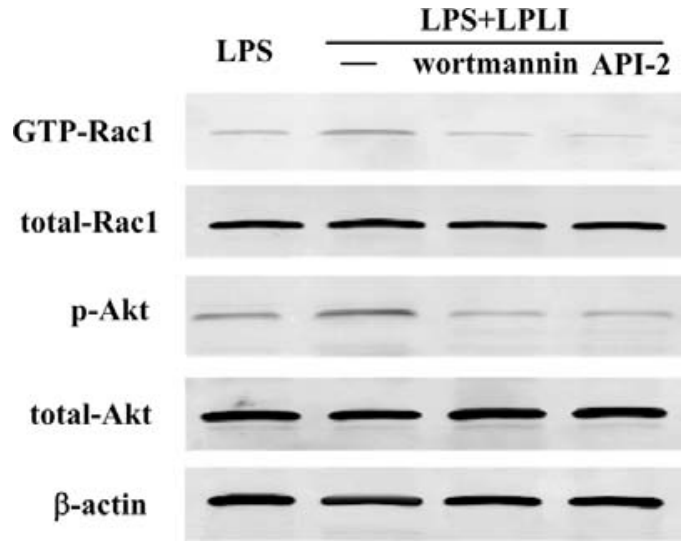

(a)

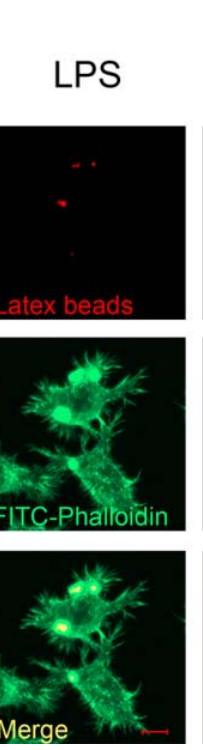

Merge

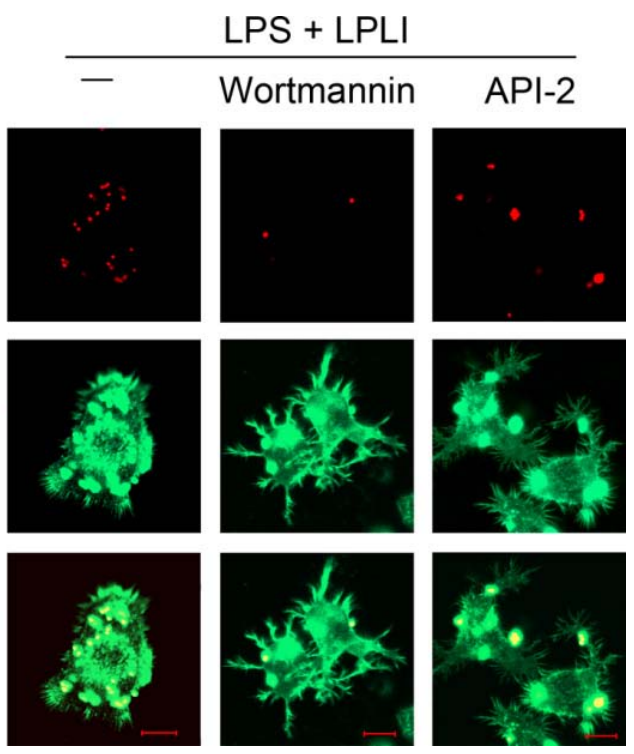

(b)

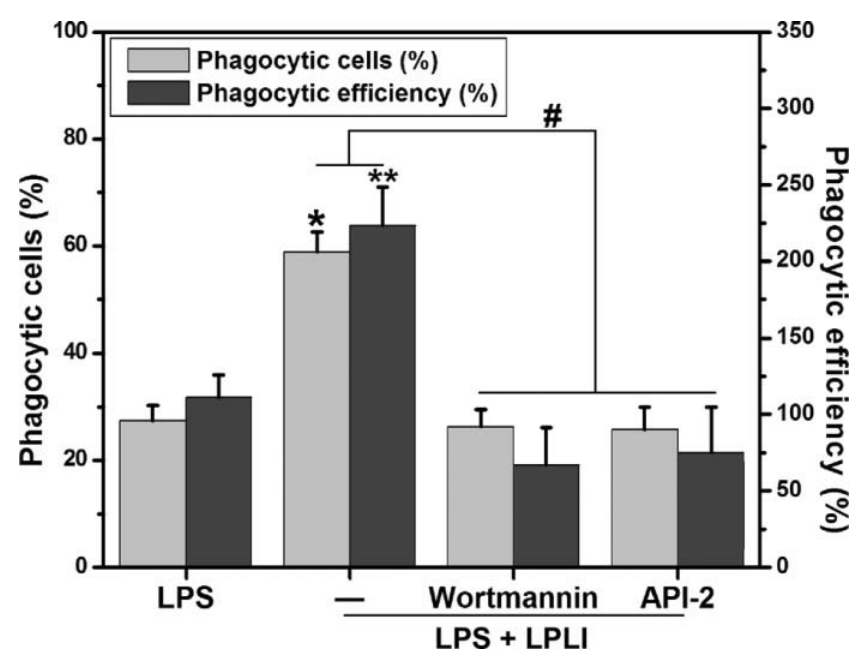

(c)

Fig. 3. LPLI-induced Rac1 activation is mediated by PI3K/Akt pathway. LPS-activated BV-2 cells pre-treated with or without wortmannin $(100 \mathrm{nM})$, or API-2 $(5 \mu \mathrm{M})$ then subjected to LPLI $\left(20 \mathrm{~J} / \mathrm{cm}^{2}\right)$ treatment. (a) Representative western blot analysis of LPLI-treated LPS-activated BV-2 cells was performed to detect GTP-Rac1, total Rac1, p-Akt (ser473) and total Akt. (b) Fluorescent images of the distinct microglial phagocytosis in BV-2 cells. Merged beads (red) and F-actin (green) are displayed in yellow (bottom). Images shown are representative of approximately 100 cells. Bar $=10 \mu \mathrm{m}$. (c) Quantification of LPLI-mediated microglial phagocytosis; and the number of microspheres taken up per BV-2 cell at 30 min was measured. $\left(n=4 ;{ }^{*} p<0.05\right.$ and ${ }^{* *} p<0.05$ vs corresponding control cells, $p<0.05$ vs indicated cells).

Understanding the mechanism and functional significance of microglial-mediated phagocytosis may lead to new neurotherapies.

LPS acts as a potent stimulator of microglia and has been used to study the activation of microglia in the pathogenesis of $\mathrm{AD}$ for $\mathrm{AD}$ treatment. Evidence from some rat models shows that microglia is activated immediately after LPS injection. Significant elevations of cluster differentiation marker CD45, glial fibrillary acidic protein (GFAP), scavenger receptor A (SRA), and Fc $\gamma$ receptor mRNA were seen after $24 \mathrm{~h} .{ }^{25}$ LPS-induced inflammation also exacerbates phosphotau pathology in rTg4510 mice. ${ }^{26}$

Much attention has been paid to therapeutic strategies aimed at controlling microglial-mediated 
neurotoxicity. LPLI is a nonthermal irradiation within the visible to near infrared range of light spectrum which has been used clinically to accelerate wound healing and reduce pain and inflammation in a variety of pathologies. ${ }^{27}$ Recently, it has been debated whether He-Ne laser light can activate a number of signaling pathways including MAPK/ERK, Src, Akt and RTK/PKCs signaling pathway. ${ }^{6,28-30}$ Nonreceptor tyrosine kinases are critical for the regulation of fundamental cellular processes including cell proliferation, adhesion, migration and survival.

We explored the role of Rac1 activation involved in LPS-activated microglia after LPLI treatment. In our experiments, we demonstrated that LPLI triggered a significant activation of Rac1 in LPSactivated BV-2 cells (see Fig. 2). Besides, the activation of Rac1 triggered by LPLI was mediated by PI3k/Akt pathway [see Fig. 3(c)]. LPLI-mediated microglia phagocytosis could significantly be suppressed by PI3K or Akt inhibitors [see Figs. 2(a) and 2(b)].

How does LPLI activate PI3K/Akt? One of the most plausible explanations is that LPLI activates PI3K through ROS. LPLI has been demonstrated to increase the level of intracellular ROS generation. ${ }^{31}$ With LPLI treatment, light is absorbed by endogenous photosensitizers (porphyrins or cytochromes) that dominantly locate at plasma membrane, mitochondria or lysosomes. The photosensitizers' activation results in ROS (1O2, O-2 and $\mathrm{H} 2 \mathrm{O} 2$ ) production. ${ }^{32}$ Intracellular oxidants could mediate the activation of PI3K. ${ }^{33}$ This hypothesis was also supported by our previous work. ${ }^{30}$

The deposition of $\mathrm{A} \beta$ in the extracellular space of the brain plays an important role in microglial activation in $\mathrm{AD}$. Although the role of microgliamediated inflammation in the pathogenesis of $\mathrm{AD}$ is obvious, microglia has been reported to mediate the clearance of $\mathrm{A} \beta$ through receptor-mediated phagocytosis, which could delay the progression of AD.

Activation of the PI3K/Akt signaling pathway has been correlated with tumor metastasis and invasion. ${ }^{34}$ Indeed, PI3K is a key signaling molecule for integrin activation and regulation of actin reorganization. ${ }^{35}$

Microglial activation is considered as a hallmark of AD. Alternatively, microglial activation is usually associated with marked increase in CD11b expression. ${ }^{36}$ Integrin CD11b/CD18 (macrophage antigen complex 1, MAC1; also known as complement receptor 3, CR3) is essential for the phagocytosis of multiple compounds and mediates the activation of phagocytes in response to a diverse set of stimuli. ${ }^{37}$ The MAC1 receptor is located on microglia, suggesting its role in neurodegeneration. In addition, previous reports indicated that MAC1 might be a key receptor for $\mathrm{A} \beta$ to activate microglia to produce extracellular superoxide, resulting in neurotoxicity. ${ }^{38}$ Conversely, others indicated that ROS was a key player in microglial activation in which ROS increased microglial expression of CD11b via NO. ${ }^{39}$ In fact, a recent study showed that activation of CD11b via inside-out signaling negatively regulated TLRtriggered proinflammatory response. ${ }^{40}$

Taken together, the current investigation demonstrates that LPLI can enhance microglial phagocytic activity through activation of PI3K/Akt pathway in LPS-induced neuroinflammation model. Although cultured mouse microglia and its treatment with etiological reagents of $\mathrm{AD}$ may not truly resemble microglia in the brain of patients, our results suggest that targeting PI3K/Akt/Rac1 signaling pathway may be an important step for the enhancement of microglial phagocytosis. Better understanding of the regulation mechanism of activated microglia may provide a therapeutic strategy to control the progression of $\mathrm{AD}$.

\section{Acknowledgments}

We are grateful to the Dr. Michiyuki Matsuda for the help with pRaichu-Rac1.

This research is supported by the National Basic Research Program of China (2011CB910402; 2010CB732602), the Program for Changjiang Scholars and Innovative Research Team in University (IRT0829) and the National Natural Science Foundation of China $(30870676 ; 30870658)$.

\section{References}

1. V. H. Perry, J. A. Nicoll, C. Holmes, "Microglia in neurodegenerative disease," Nat. Rev. Neurol. 6, 193-201 (2010).

2. L. Minati, T. Edginton, M. G. Bruzzone, G. Giaccone, "Current concepts in Alzheimer's disease: A multidisciplinary review," Am. J. Alzheimers Dis. Other Demen. 24, 95-121 (2009).

3. G. A. Garden, T. Moller, "Microglia biology in health and disease," J. Neuroimmune Pharmacol. 1, 127-137 (2006). 
4. A. K. Stalder, A. Pagenstecher, N. C. Yu, C. Kincaid, C. S. Chiang, M. V. Hobbs, F. E. Bloom, I. L. Campbell, "Lipopolysaccharide-induced IL-12 expression in the central nervous system and cultured astrocytes and microglia," J. Immunol. 159, 1344-1351 (1997).

5. M. L. Block, L. Zecca, J. S. Hong, "Microgliamediated neurotoxicity: Uncovering the molecular mechanisms," Nat. Rev. Neurosci. 8, 57-69 (2007).

6. N. Ben-Dov, G. Shefer, A. Irintchev, A. Wernig, U. Oron, O. Halevy, "Low-energy laser irradiation affects satellite cell proliferation and differentiation in vitro," Biochim. Biophys. Acta 1448, 372-380 (1999).

7. F. Wang, T. S. Chen, D. Xing, J. J. Wang, Y. X. $\mathrm{Wu}$, "Measuring dynamics of caspase-3 activity in living cells using FRET technique during apoptosis induced by high fluence low-power laser irradiation," Lasers Surg. Med. 36, 2-7 (2005).

8. G. Shefer, I. Barash, U. Oron, O. Halevy, "Lowenergy laser irradiation enhances de novo protein synthesis via its effects on translation-regulatory proteins in skeletal muscle myoblasts," Biochim. Biophys. Acta 1593, 131-139 (2003).

9. D. Hawkins, N. Houreld, H. Abrahamse, "Low level laser therapy (LLLT) as an effective therapeutic modality for delayed wound healing," Ann. N.Y. Acad. Sci. 1056, 486-493 (2005).

10. C. E. de Araujo, M. S. Ribeiro, R. Favaro, D. M. Zezell, T. M. Zorn, "Ultrastructural and autoradiographical analysis show a faster skin repair in He-Ne laser-treated wounds," J. Photochem. Photobiol. B 86, 87-96 (2007).

11. L. Zhang, D. Xing, D. Zhu, Q. Chen, "Low-power laser irradiation inhibiting Abeta25-35-induced PC12 cell apoptosis via PKC activation," Cell Physiol. Biochem. 22, 215-222 (2008).

12. H. Zhang, S. Wu, D. Xing, "Inhibition of Abeta(2535)-induced cell apoptosis by Low-power-laser-irradiation (LPLI) through promoting Akt-dependent YAP cytoplasmic translocation," Cell. Signal 24, 224-232 (2012).

13. L. Van Aelst, C. D'Souza-Schorey, "Rho GTPases and signaling networks," Genes Dev. 11, 2295-2322 (1997).

14. W. E. Allen, G. E. Jones, J. W. Pollard, A. J. Ridley, "Rho, Rac and Cdc42 regulate actin organisation and cell adhesion in macrophages," J. Cell Sci. 110, 707-720 (1997).

15. D. Cox, P. Chang, Q. Zhang, P. G. Reddy, G. M. Bokoch, S. Greenberg, "Requirements for both rac1 and cdc42 in membrane ruffling and phagocytosis in leukocytes," J. Exp. Med. 186, 1487-1494 (1997).

16. X. Gao, T. Chen, D. Xing, F. Wang, Y. Pei, X. Wei, "Single cell analysis of PKC activation during proliferation and apoptosis induced by laser irradiation," J. Cell. Physiol. 206, 441-448 (2006).

17. Y. Zhang, D. Xing, L. Liu, "PUMA promotes Bax translocation by both directly interacting with Bax and by competitive binding to Bcl-X L during UVinduced apoptosis," Mol. Biol. Cell. 20, 3077-3087 (2009).

18. X. D. Pan, Y. G. Zhu, N. Lin, J. Zhang, Q. Y. Ye, H. P. Huang, X. C. Chen, "Microglial phagocytosis induced by fibrillar beta-amyloid is attenuated by oligomeric beta-amyloid: Implications for Alzheimer's disease," Mol. Neurodegener. 6, 45 (2011).

19. J. M. Blander, R. Medzhitov, "Regulation of phagosome maturation by signals from toll-like receptors," Science 304, 1014-1018 (2004).

20. T. Joneson, M. McDonough, D. Bar-Sagi, L. Van Aelst, "RAC regulation of actin polymerization and proliferation by a pathway distinct from Jun kinase," Science, 274, 1374-1376 (1996).

21. C. Albertinazzi, A. Cattelino, I. de Curtis, "Rac GTPases localize at sites of actin reorganization during dynamic remodeling of the cytoskeleton of normal embryonic fibroblasts," J. Cell. Sci. 112(Pt 21), 3821-3831 (1999).

22. F. Castellano, P. Montcourrier, P. Chavrier, "Membrane recruitment of Rac1 triggers phagocytosis," J. Cell. Sci. 113 (Pt 17), 2955-2961 (2000).

23. K. Volling, A. Thywissen, A. A. Brakhage, H. P. Saluz, "Phagocytosis of melanized Aspergillus conidia by macrophages exerts cytoprotective effects by sustained PI3K/Akt signalling," Cell. Microbiol. 13, 1130-1148 (2011).

24. N. Michio, K. Masahiro, M. Michiyuki, N. Shigekazu, "Spatiotemporal activation of Rac1 for engulfment of apoptotic cells," Proc. Natl. Acad. Sci. USA 105, 9198-9203 (2008).

25. D. L. Herber, J. L. Maloney, L. M. Roth, M. J. Freeman, D. Morgan, M. N. Gordon, "Diverse microglial responses after intrahippocampal administration of lipopolysaccharide," Glia 53, 382-391 (2006).

26. D. C. Lee, J. Rizer, M. L. Selenica, P. Reid, C. Kraft, A. Johnson, L. Blair, M. N. Gordon, C. A. Dickey, D. Morgan, "LPS-induced inflammation exacerbates phospho-tau pathology in rTg4510 mice," $J$. Neuroinflammation 7, 56 (2010).

27. A. Schindl, M. Schindl, H. Pernerstorfer-Schon, K. Kerschan, R. Knobler, L. Schindl, "Diabetic neuropathic foot ulcer: Successful treatment by lowintensity laser therapy," Dermatology 198, 314-316 (1999).

28. L. Zhang, D. Xing, X. Gao, S. Wu, "Low-power laser irradiation promotes cell proliferation by 
activating PI3K/Akt pathway," J. Cell. Physiol. 219, 553-562 (2009).

29. X. Gao, T. Chen, D. Xing, F. Wang, Y. Pei, X. Wei, "Single cell analysis of PKC activation during proliferation and apoptosis induced by laser irradiation," J. Cell. Physiol. 206, 441-448 (2006).

30. J. Zhang, D. Xing, X. Gao, "Low-power laser irradiation activates Src tyrosine kinase through reactive oxygen species-mediated signaling pathway," J. Cell. Physiol. 217, 518-528 (2008).

31. S. Matsui, Y. Tsujimoto, K. Matsushima, "Stimulatory effects of hydroxyl radical generation by Ga-Al-As laser irradiation on mineralization ability of human dental pulp cells," Biol. Pharm. Bull. 30, 27-31 (2007).

32. R. Lavi, A. Shainberg, H. Friedmann, V. Shneyvays, O. Rickover, M. Eichler, D. Kaplan, R. Lubart, "Low energy visible light induces reactive oxygen species generation and stimulates an increase of intracellular calcium concentration in cardiac cells," $J$. Biol.Chem. 278, 40917-40922 (2003).

33. D. J. Kemble, G. Sun, "Direct and specific inactivation of protein tyrosine kinases in the Src and FGFR families by reversible cysteine oxidation," Proc. Natl. Acad. Sci. USA 106, 5070-5075 (2009).

34. K. Jiang, J. Sun, J. Cheng, J. Y. Djeu, S. Wei, S. Sebti, "Akt mediates Ras downregulation of RhoB, a suppressor of transformation, invasion, and metastasis," Mol. Cell. Biol. 24, 5565-5576 (2004).
35. Y. Qian, L. Corum, Q. Meng, J. Blenis, J. Z. Zheng, X. Shi, D. C. Flynn, B. H. Jiang, "PI3K induced actin filament remodeling through Akt and p70S6K1: Implication of essential role in cell migration," Am. J. Physiol. Cell. Physiol. 286, C153-163 (2004).

36. H. Akiyama, P. L. McGeer, "Brain microglia constitutively express beta-2 integrins," J. Neuroimmunol. 30, 81-93 (1990).

37. V. Le Cabec, S. Carreno, A. Moisand, C. Bordier, I. Maridonneau-Parini, "Complement receptor 3 (CD11b/CD18) mediates type I and type II phagocytosis during nonopsonic and opsonic phagocytosis, respectively," J. Immunol. 169, 2003-2009 (2002).

38. D. Zhang, X. Hu, L. Qian, S. H. Chen, H. Zhou, B. Wilson, D. S. Miller, J. S. Hong, "Microglial MAC1 receptor and PI3K are essential in mediating betaamyloid peptide-induced microglial activation and subsequent neurotoxicity," J. Neuroinflammation $\mathbf{8}$, 3 (2011).

39. A. Roy, A. Jana, K. Yatish, M. B. Freidt, Y. K. Fung, J. A. Martinson, K. Pahan, "Reactive oxygen species up-regulate CD11b in microglia via nitric oxide: Implications for neurodegenerative diseases," Free Radic. Biol. Med. 45, 686-699 (2008).

40. C. Han, J. Jin, S. Xu, H. Liu, N. Li, X. Cao, "Integrin CD11b negatively regulates TLR-triggered inflammatory responses by activating Syk and promoting degradation of MyD88 and TRIF via Cbl-b," Nat. Immunol. 11, 734-742 (2010). 